\title{
Kosteneinsparungen dank Änderung der Preisgestaltung von Originalpräparaten
}

Andreas Keusch

Medizinischer Berater

Interessenverbindungen: Die Veröffentlichung dieses Artikels ist für den Autor mit keinen persönlichen Interessenverbindungen verknüpft.

\footnotetext{
* Die in diesem Text verwendete männliche Form gilt für beide Geschlechter und dient einzig der besseren Lesbarkeit.
}

Korrespondenz:

Dr. med. vet. Andreas Keusch MEDVICE

Sonnenhof 1

CH-8808 Pfäffikon SZ

Tel. 0554429268

medvice@bluewin.ch

\section{Ausgangslage}

Ein neues Arzneimittel (Originalpräparat) ist wirtschaftlich von Bedeutung, wenn es in der medizinischen Behandlung einen therapeutischen Fortschritt erzielt und die Kosten von der Grundversicherung getragen werden. Eine Kostenübernahme erfolgt nach Aufnahme in die Spezialitätenliste (SL) [1]. Zur Beurteilung werden Wirksamkeit, Wirtschaftlichkeit und Zweckmässigkeit des Präparates herangezogen (siehe Tabelle) [1,2]. Die Wirksamkeit stellt aufgrund der vorliegenden Zulassung der Swissmedic selten einen Grund zur Beanstandung dar. Als wirtschaftlich gilt das Medikament, wenn die indizierte Heilwirkung im Vergleich zu Arzneimitteln desselben Indikationsbereiches mit möglichst geringem finanziellem Aufwand gewährleistet und im Auslandspreisvergleich akzeptiert wird. Für die Beurteilung der Zweckmässigkeit hinsichtlich unerwünschter Wirkungen und Komplikationen als zusätzlicher Kostenfaktor einer Therapie stehen den Behörden häufig nur die klinischen Daten des Zulassungsgesuchs zur Verfügung.

Da jeder Hersteller seine Substanz mit einem Patent schützt, hängt der wirtschaftliche Erfolg wesentlich von der vor Generika geschützten Vermarktungsdauer ab, die nach der Entwicklungs- und Registrierungsphase noch übrigbleibt: ca. 8-12 Jahre (exkl. Schutzzertifikat) $[3,4]$. Die stetig höheren Qualitätsanforderungen der klinischen Studien zum Schutze des Studienteilnehmers* lassen die Entwicklungszeit jedoch stets weiter ansteigen. Dies resultiert im Zulassungsgesuch in einer minimal notwendigen, aber ausreichenden Abklärung der Arzneimittelsicherheit. Der Patientenpool wird so optimiert, dass das Risiko schwerwiegender Nebenwirkungen oder Todesfälle, die eine Zulassung um Jahre verzögern oder sogar verhindern, möglichst gering ausfällt. Ein optimales Nutzen-Risiko-Profil stellt bei der Preisfestlegung einen immensen Vorteil dar. Die Gesamtanzahl behandelter Studienpatienten fällt im Vergleich zum Marktpotential mit seinen Patienten, die häufig an Begleiterkrankungen leiden und Co-Medikationen einnehmen, jedoch sehr gering aus, weshalb sich das tatsächliche Nutzen-Risiko-Profil erst im Verlaufe der Vermarktung (Langzeitanwendung) bestimmen lässt. Ironischerweise werden aber bereits Innovationszuschläge zugesprochen, obwohl zum Zeitpunkt der Markteinführung die tatsächliche Inzidenz an bisher noch unbekannten und bekannten Nebenwirkungen und Komplikationen in der Fachinformation erst zu 10-20\% bekannt ist! Es sind aber gerade die Nebenwirkungen

\section{Economies de coûts grâce à la modification de la tarification des préparations originales}

La spirale des prix qui progresse à chaque nouvelle admission de préparations originales peut être ralentie en n'accordant plus de primes à l'innovation lors de la première admission sur la liste des spécialités. L'admission sur la liste des spécialités s'effectue au prix de la thérapie actuelle qui a fait ses preuves au plan médical et économique. Les primes à l'innovation, y compris la prolongation de la protection des brevets, ne sont accordées en guise de rémunération appropriée et d'indemnisation des frais pour une activité de recherche et de développement innovante que si la demande d'augmentation de prix démontre, dans des conditions d'utilisation pratique et prolongée, un avantage significatif en termes d'efficacité et de sécurité par rapport à la thérapie standard. Les frais de traitement générés par la prescription de la préparation originale doivent représenter un avantage financier parallèlement au progrès thérapeutique. Cela évite à la collectivité d'avoir à supporter les coûts de la première phase de commercialisation expérimentale, à l'occasion de laquelle le profil risque-utilité déterminant pour la pratique est encore déterminé. La compréhension générale de la sécurité des médicaments est encouragée, les attentes généralement trop élevées à l'égard du nouveau médicament générées par les médias sont tempérées et la confiance et la compliance du patient dans les recommandations de soins de son médecin sont renforcées.

und Komplikationen, die mit zusätzlichen therapeutischen Massnahmen (z. B. Co-Medikationen) die Behandlung unnötig verteuern und die bei der Preisfestlegung primär in Aussicht gestellten Kosteneinsparungen der Therapie mindern oder sogar übertreffen. 
Bei der Überprüfung der Aufnahmebedingungen in die SL stehen die Kriterien der Wirtschaftlichkeit im Vordergrund. Die Zweckmässigkeit scheint zweitrangig zu sein.

So begünstigt die aktuelle Politik zur Preisfestlegung eine Aufnahme in die SL anhand einer limitierten Datenlage, die eine Zulassung rechtfertigt, sich aber im Vergleich zu erwiesenen sicheren Standardtherapien hinsichtlich Wirksamkeit, Sicherheit und Verträglichkeit erst noch in der täglichen Verordnungspraxis und Langzeitanwendung mit grosser $\mathrm{Pa}$ tientenzahl beweisen muss [5,6]. Die ersten Verordnungsjahre stellen vor allem hinsichtlich Sicherheit gen für den Hersteller in geringem Rahmen halten, da er den Innovationszuschlag zur Deckung der Kosten für Forschung und Entwicklung bereits bei der Markteinführung erhielt. Das Risiko und die Kosten tragen der Patient bzw. die Allgemeinheit. Das Vertrauensverhältnis zwischen Arzt und Patient kann aufgrund der ungerecht hohen, medial induzierten Erwartungshaltung des Patienten empfindlich gestört werden. Die Preisspirale dreht sich ständig weiter nach oben, da jedes neu eingeführte Originalpräparat im Vergleich zu dem zuletzt in die SL aufgenommenen Präparat Anspruch auf Innovationszuschläge erhebt.

\section{Eine nachgewiesene Zweckmässigkeit nach Markteinführung als Ergebnis qualitativ hochstehender Forschung, Entwicklung und Marktüberwachung wird nicht honoriert}

und Verträglichkeit noch eine experimentelle Testphase in der Vermarktung dar, die kostenmässig von unserem Gesundheitswesen getragen wird.

Höchstpreise werden verlangt für durchschnittliche Qualität, resp. Forschung. Zwei Beispiele sind Vioxx $^{\circledR}$ und Zelmac ${ }^{\circledast}$, die aufgrund ihres nach der Zulassung festgestellten ungünstigen Nutzen-Risiko-Profils sogar vom Markt genommen werden mussten. Der wirtschaftliche Schaden dürfte sich dank der erzielten hohen Verkaufspreise und anfänglichen Markterfol-

\section{Senkung der Arzneimittelkosten}

Die aktuelle Senkung der Arzneimittelkosten beruht im Wesentlichen auf der Beurteilung der Wirtschaftlichkeit. Der Höchstpreis wird zum Zeitpunkt der Aufnahme in die SL gewährt. Danach ist bei der Überprüfung der Aufnahmebedingungen in die SL nur noch mit Preissenkungen zu rechnen. Dem Hersteller wird so kein zusätzlicher Anreiz geboten, sich nach der Zulassung wesentlich für die qualitative Abklärung/ Sicherung des Nutzen-Risiko-Profils einzusetzen. Mar-

\section{Tabelle \\ Definition der Kriterien für die Aufnahme in die Spezialitätenliste \\ Wirksamkeit \\ Das BAG stützt sich für die Beurteilung der Wirksamkeit auf die Unterlagen, die für die Registrierung durch die Swissmedic massgebend waren. Es kann weitere Unterlagen verlangen. ${ }^{1}$}

\section{Zweckmässigkeit}

Die Zweckmässigkeit eines Arzneimittels in Bezug auf seine Wirkung und Zusammensetzung wird nach klinisch-pharmakologischen und galenischen Erwägungen, nach unerwünschten Wirkungen sowie nach der Gefahr missbräuchlicher Verwendung beurteilt.'

Das BAG stützt sich für die Beurteilung der Zweckmässigkeit auf die Unterlagen, die für die Zulassung durch die Swissmedic massgebend waren. Es kann weitere Unterlagen verlangen. ${ }^{1}$

\section{Wirtschaftlichkeit}

Ein Arzneimittel gilt als wirtschaftlich, wenn es die indizierte Heilwirkung mit möglichst geringem finanziellem Aufwand gewährleistet. ${ }^{1}$ Für die Beurteilung der Wirtschaftlichkeit eines Arzneimittels werden berücksichtigt:

- Dessen Fabrikabgabepreise im Ausland;

- Dessen Wirksamkeit im Verhältnis zu anderen Arzneimitteln gleicher Indikation oder ähnlicher Wirkungsweise;

- Dessen Kosten pro Tag oder Kur im Verhältnis zu den Kosten von Arzneimitteln gleicher Indikation oder ähnlicher Wirkungsweise;

- Bei einem Arzneimittel im Sinne von Artikel 31 Absatz 2 Buchstaben a und b ein Innovationszuschlag für die Dauer von höchstens 15 Jahren; in diesem Zuschlag sind die Kosten für Forschung und Entwicklung angemessen zu berücksichtigen. ${ }^{1}$

Die Wirtschaftlichkeit wird aufgrund des Vergleichs mit anderen verwendungsfertigen Arzneimitteln und der Preisgestaltung im Ausland beurteilt. Der Auslandspreisvergleich erfolgt summarisch, wenn er mangels Zulassung in den Vergleichsländern im Zeitpunkt des Gesuchs um Aufnahme nicht oder nur unvollständig vorgenommen werden kann. ${ }^{2}$

${ }^{1}$ KLV [1] / 2 KVV [2] 
ketingstrategien wie z.B. unerlaubte indirekte Werbung über Selbsthilfegruppen, News in Print- und elektronischen Medien werden bevorzugt und gezielt gefördert. Die Forschungsbestrebungen werden im Wesentlichen auf Neuentwicklungen fokussiert, die mit minimalem Aufwand eine Zulassung erhalten und gleichzeitig Innovationszuschläge rechtfertigen. Nachweisliche Zweckmässigkeit nach Markteinführung als Belohnung für qualitativ hochstehende Forschung, Entwicklung und Marktüberwachung werden nicht honoriert. Ein Paradigmenwechsel hin zu möglichen Preiserhöhungen könnte diese Haltung jedoch ändern und gleichzeitig die Arzneimittelqualität bei sinkenden Gesamtkosten erhöhen.

Diesem Ansatzpunkt liegt folgende Hypothese zugrunde: Zum Zeitpunkt der Markteinführung eines Originalpräparates soll der Preis mit der medizinisch aktuell gültigen Standardtherapie vergleichbar sein. Erst wenn das Medikament sich wirtschaftlich hinsichtlich Effektivität und Sicherheit in der täglichen Verordnungspraxis nach mehrjährigem Einsatz (Langzeiterfahrung) mit medizinisch wirtschaftlichen (ökonomischen) Kosteneinsparungen bewährt, darf ein Innovationszuschlag zu einer Preiserhöhung führen.
Diese müssen im Vergleich zur Standardtherapie eine signifikant ökonomisch nachweisbare Kosteneinsparung belegen. Der gewährte Innovationsbonus darf die erzielten Kosteneinsparungen nachträglich nicht ausgleichen. Trotz Preiserhöhung muss noch immer ein wirtschaftlicher Vorteil resultieren. Werden zur Evaluation der Zweckmässigkeit Ergebnisse von Langzeitstudien von $\geq 5$ Jahren benötigt, kann eine Zwischenanalyse nach Hälfte der Beobachtungsdauer zu einer ersten, reversiblen Teilerhöhung des Preises führen. Da der Patentschutz vor einer allfälligen Preiserhöhung weiter abläuft, soll er bei Erteilung des Innovationszuschlages bei Präparaten in der Akuttherapie automatisch um 5 Jahre, bei Präparaten zur Langzeittherapie/Prophylaxe um 5 bis max. 10 Jahre verlängert werden.

\section{Vorteile}

\section{Kostensenkung}

Mit Aufnahme des Originalpräparates in die SL erfolgt die Einführung eines neuen, wirksamen und relativ sicheren Heilmittels in der zugelassenen Indikation. Hersteller, Patient und Arzt sind sich aber deutlicher

\section{Die mit jeder Neuaufnahme von Originalpräparaten steigenden Kosten können reduziert werden, wenn bei der Erstaufnahme in die Spezialitätenliste keine Innovationszuschläge mehr erteilt werden}

Eine gleichzeitig gewährte Verlängerung des Patentschutzes belohnt die Forschungsinvestitionen des Herstellers und schützt diesen vor Generika. Diese Massnahme bewirkt eine Senkung der Arzneimittelkosten und verlangsamt die allgemeine Verteuerung dieser, da Forschung und Entwicklung erst nach nachweislich erbrachten Leistungskriterien belohnt werden.

\section{Umsetzung}

Die Grundvoraussetzungen für eine Aufnahme in die SL bleiben bestehen. Im Gegensatz zur aktuellen Preisgestaltung wird bei der Erstaufnahme kein Innovationszuschlag mehr gewährt. Die Aufnahme «per se» gilt als Bestätigung der Wirksamkeit, Zweckmässigkeit und Wirtschaftlichkeit des Originalpräparates. Als Preisreferenz dient eine nach Evidence-Based-Medecine-Kriterien erhobene Standardtherapie, die mindestens alle 4 Jahre neu evaluiert werden muss. Als Folge bleibt es dem Hersteller überlassen, ob er frühestens 4 Jahre nach Erstaufnahme oder spätestens 2 Jahre vor Patentablauf eine Preiserhöhung im Rahmen eines wie bisher erteilten Innovationszuschlages beantragt. Das Dossier beinhaltet Daten aus den klinisch kontrollierten Studien der Phasen I bis IV, den Marktbeobachtungsstudien und der «Postmarketing Surveillance». bewusst, dass das Nutzen-Risiko-Profil erst noch in der täglichen Verordnungspraxis bestätigt werden muss. Preiserhöhungen, die das Gesundheitswesen belasten, werden nur noch zugelassen, wenn das Heilmittel sich im Vergleich zur Standardtherapie nachweislich als sicher und effektiv erweist. Die Erteilung von Innovationszuschlägen bei der Erstaufnahme in die SL und die damit automatisch verbundene Verteuerung der Behandlungskosten können so vermieden werden. Überteuerte Präparate, die anfänglich als sicher und innovativ erscheinen, mit fortschreitender Anwendung die Erwartungen aber nicht erfüllen, eventuell sogar vom Markt genommen werden müssen, belasten die Gesamtkosten nicht mehr im selben Ausmasse wie bisher, da sie gleich viel wie die Standardtherapie kosten. Steigende Arzneimittelpreise korrelieren in Zukunft nur noch mit nachweislich erzielten Kosteneinsparungen in der zugelassenen Indikation.

\section{Förderung der Arzneimittelsicherheit}

Die minimale Qualitätssicherung als Grundlage für die Erstaufnahme in die SL reicht nicht mehr aus, um einen Innovationszuschlag zu erhalten. Der Hersteller muss in der ersten, noch experimentellen Testphase der Vermarktung daran interessiert sein, das genaue 
Nutzen-Risiko-Profil in der täglichen Praxis- und Langzeitanwendung zu erheben. Der korrekten Erfassung von Nebenwirkungen und Komplikationen im Rahmen der «Postmarketing Surveillance» wird zukünftig allgemein grössere Bedeutung beigemessen, da diese im Falle eines Preiserhöhungsgesuches nun im zentralen Interesse des Herstellers steht. Klinische Studien der Phase-IV erlangen ihre eigentliche Bedeutung zur Erhebung des Nutzen-Risiko-Profiles zurück und würden zur Erhebung des Sicherheitsprofils wieder vermehrt durchgeführt werden [6].

Von Beginn an qualitativ hochwertige Forschung und Entwicklung kann sich, im Vergleich zur Standardtherapie, im Verlaufe der ersten Verordnungsjahre als wirkliche medizinische Innovation mit wirtschaftlich belegbaren Einsparungen auszeichnen. Die Forschung wird so von einem minimalen, für eine $\mathrm{Zu}$ lassung notwendigen auf ein qualitativ optimaleres, für einen Innovationszuschlag (inkl. Patentschutzverlängerung) benötigtes Niveau angehoben.

\section{Förderung Vertrauensverhältnis Arzt-Patient}

Die vom Hersteller über die Medien und Selbsthilfegruppen generierte Erwartungshaltung bei den Anwendern «Das Neueste ist das Beste ist das Teuerste», wird auf ein vernünftiges Niveau zurückgestuft. Dies ermöglicht dem Arzt vermehrt die Verordnung des für den Patienten am besten geeigneten Präparates, da der medial erzeugte Verordnungsdruck abnimmt. Die Gefahr, einen Patienten bei Problemen als schwierig oder als Simulanten einzustufen, reduziert sich, da die Erhebung des Nutzen-Risiko-Profils der Behandlung gefördert wird. Dies verbessert einerseits die Compliance des Patienten gegenüber seiner Behandlung, andererseits stärkt es das Vertrauen in den Arzt, da der Abklärung der Sicherheit und Verträglichkeit grössere Aufmerksamkeit geschenkt wird. Erst Präparate mit Preiserhöhung ermöglichen im Vergleich zur Standardtherapie die Aussagen der Hersteller wie «wirksamer», «sicherer», «praktisch keine Nebenwirkungen» und stellen für Arzt und Patient so einen glaubwürdigen Fortschritt in der medizinischen Behandlung dar.

\section{Fazit}

Die sich mit jeder Neuaufnahme von Originalpräparaten nach oben drehende Preisspirale kann abgebremst werden, wenn bei der Erstaufnahme in die SL keine Innovationszuschläge mehr erteilt werden. Die Aufnahme in die SL erfolgt zum Preis der aktuell medizinisch und wirtschaftlich erwiesenen Standardtherapie. Innovationszuschläge inkl. Patentschutzverlängerung werden als angemessene Belohnung und Kostenabgeltung für innovative Forschung und Entwicklung erst erteilt, wenn das Preiserhöhungsgesuch im Vergleich zur Standardtherapie unter Praxisbedingungen und Langzeitanwendung einen nachweisbar signifikanten Vorteil hinsichtlich Wirksamkeit und Sicherheit belegen kann. Die mit der Verordnung des Originalpräparates erzielten Behandlungskosten müssen einen finanziellen Vorteil bei gleichzeitig therapeutischem Fortschritt darstellen. Dies verhindert, dass die Allgemeinheit die Kosten der ersten experimentellen Vermarktungsphase, bei der das praxisrelevante Nutzen-Risiko-Profil des Präparates noch ermittelt wird, zu tragen hat. Das Allgemeinverständnis gegenüber der Arzneimittelsicherheit wird gefördert, die allgemein in den Medien generierte zu hohe Erwartungshaltung an das neue Medikament gesenkt und das Vertrauen und die Compliance des Patienten in die Behandlungsempfehlung seines Arztes gestärkt.

\section{Literatur}

1 SR 832.112.31 Verordnung des EDI über Leistungen in der obligatorischen Krankenpflegeversicherung (KLV).

2 SR 832.102 Verordnung vom 27. Juni 1995 über die Krankenversicherung (KVV).

3 SR 232.14 Bundesgesetz vom 25. Juni 1954 über die Erfindungspatente (Patentgesetz, PatG).

4 SR 232.141 Verordnung vom 19. Oktober 1977 über die Erfindungspatente (Patentverordnung, PatV).

5 Berger M, Mühlhauser I. Arzneimittelsicherheit: Wirksamkeit von Medikamenten muss auch nach Zulassung geprüft werden. Dtsch Ärztebl. 2000;97(4):154-6.

6 Wood AJJ. A proposal for radical changes in the drug-approval process. N Engl J Med. 2006;355:618-23. 